\title{
Enhancement Of Satellite Image Resolution With Moving Objects
}

\author{
A. Abirami ${ }^{1}$, N. Akshaya ${ }^{2}$, D. Poornakala ${ }^{3}$, D. Priyanka ${ }^{4}$, C. Ram kumar ${ }^{5}$ \\ 1,2,3,4 UG Students, SNS College of Engineering, Coimbatore - 641107. \\ ${ }^{5}$ Assistant professor, SNS College of Engineering, Coimbatore - 641107.
}

\begin{abstract}
Satellite images are being used in many applications like Meteorology, Agriculture, Geology, Forestry, Landscape, Biodiversity, Planning, Education, Regional, Seismology and oceanography. The Enhanced satellite images make diagnostic details more obvious. The Image Enhancement is the main technique for improving the resolution and visual appearance of the image. One of the major issues in Image Enhancement is Wavelet Transform. The Wavelet Transform is the method which decomposes an image into a set of basic functions called Wavelets. These basis functions are limited in duration and are inherently local. A Resolution Enhancement technique is based on the Interpolation of the high-frequency subbands obtained by Discrete Wavelet Transforms (DWT). Bicubic interpolation is used as a intermediate stage for estimating high frequency components and it is more sophisticated than the nearest neighbor and bilinear techniques. The proposed technique has the advantages of superior resolution, sharper image and smoother edges. The PSNR improvement of the proposed technique is up to $7.19 \mathrm{~dB}$.
\end{abstract}

Keywords - Image resolution, Edge detection, transforms, cycle spinning

\section{Introduction}

Image enhancement is a method of improving the definition of a video picture by a computer program, which reduces the lowest gray values to black and the highest to white, for the pictures from microscopes, surveillance cameras, and scanners. Improvement of the quality of a picture, with the aid of a computer, by giving it higher contrast or making it less blurred or less noisy. Image enhancement techniques can be divided into two broad categories are Spatial domain methods, which operate directly on pixels and frequency domain methods, which operate on the Fourier transform of an image.

Interpolation in image processing is a method to increase the number of pixels in a digital image. Interpolation has been widely used in many image processing applications, such as facial reconstruction, multiple description coding, and image resolution enhancement .The interpolation-based image resolution enhancement has been used for a long time and many interpolation techniques have been developed to increase the quality of this task.

Wavelets are also playing a significant role in many image processing applications. The 2-D wavelet decomposition of an image is performed by applying the 1-D discrete wavelet transform (DWT) along the rows of the image first, and then the results are decomposed along the columns. This operation results in four decomposed sub-band images referred to low-low (LL), low-high (LH), high-low (HL), and high-high (HH). The frequency components of those sub-bands cover the full frequency spectrum of the original image. A satellite image resolution enhancement technique is based on the interpolation of the high-frequency subband obtained by discrete wavelet transform (DWT) and the input image. This resolution enhancement technique uses DWT to decompose the input image into different subband. Then, the high-frequency subband images and the input low resolution image have been interpolated, followed by combining all these images to generate a new resolution enhanced image.

\section{Literature Survey}

\subsection{Cycle Spinning And Edge Modelling}

Recursive Cycle Spinning (RCS) was employed to avoid the artifacts. This method can get the better visual effect and PSNR value compared with the methods like wavelet image de-noising using the Recursive Cycle Spinning ${ }^{[16]}$. Artifact is a classic signal interpolation problem and conventional approaches such as zeroorder interpolation (sample-and-hold) cause severe pixelation impairments while bilinear and spline interpolation invariably result in undesirable levels of smoothing across salient edges. Recently several efforts in the field have utilized wavelet-domain methodologies with the intention of overcoming some of the problems associated with conventional treatment ${ }^{[2]}$. A common feature of these algorithms is the assumption that the lowresolution (LR) image to be enhanced is the low-pass filtered sub-band of a high-resolution (HR) image which has been subjected to a decimated wavelet transform. 


\subsection{Edge Detection For Images}

Edge detection is a fundamental tool in image processing, machine vision and computer vision, particularly in the areas of feature detection and feature extraction ${ }^{[12]}$. which aim at identifying points in a digital image at which the image brightness changes sharply or, more formally, has discontinuities. Edges are significant local changes of intensity in an image ${ }^{[14]}$. Edges typically occur on the boundary between two different regions in an image.

An edge may be loosely defined as a local discontinuity in the pixel value that exceeds a given threshold. There are many steps in edge detection which are as follows:

Smoothing: Suppress as much noise as possible, without destroying the true edges.

Enhancement: Apply a filter to enhance the quality of the edges in the image (sharpening).

Detection: Determine which edge pixels should be discarded as noise and which should be retained (usually, thresholding provides the criterion used for detection).

Localization: Determine the exact location of an edge (sub-pixel resolution might be required for some applications, that is, estimate the location of an edge to better than the spacing between pixels). Edge thinning and linking are usually required in this method.

\subsection{Image Resolution Enhancement Using Inter-Subband Correlation In Wavelet Domain}

Conventional image resolution enhancement methods, such as bilinear and bicubic interpolation methods may generate false information and blurred images because they do not utilize any information relevant to edges in the original image ${ }^{[13]}$. Wavelet-based methods enhanced the image resolution by estimating the preserved high frequency information from the given images ${ }^{[7]}$. They were based on the assumption that the image to be enhanced was the low frequency subband among wavelet-transformed subband of the original one and the target is to estimate the high frequency subband of wavelet transform, so that a resolution enhanced image can be obtained ${ }^{[1]}$. Because the analysis filter bank used in the wavelet transform has a poor frequency characteristic such as wide transition region, some information on the high-frequency band has remained in the low frequency band. The phase - shifting matrix was first derived by XinLi, for saving the computational complexity of LBS method ${ }^{[10]}$. It showed that two sub-bands of a two-channel perfect reconstruction filter bank was linked by a unique phase-shifting matrix. Since it is customary to put the center of an odd symmetric filter at 0 , for odd length biorthogonal filters, the sample positions in the low-frequency band and the high frequency band are alternative, which means even-phase for low frequency band and odd phase for high frequency band.

\subsection{CYCLE SPINNING}

The decimated wavelet transform is not shift-invariant and as a result, distortion of wavelet coefficients, due to quantization of coefficients in compression applications or non-exact estimation of highfrequency coefficients in resolution enhancement applications (including zero padding of coefficients as in WZP), introduces cyclostationarity into the image which manifests itself as ringing in the neighborhood of discontinuities.

Cycle-spinning (CS) is an effective method against ringing when used for de-noising purposes in the wavelet domain and also for reducing ringing and increasing the perceptual quality of compressed images ${ }^{[16]}$. CS method aims to approximate shift-invariant statistics by averaging out the cyclostationarity ${ }^{[1]}$. CS can also be applied as a post-processing operation after decompression results in significant improvements in the framework of JPEG and JPEG2000 image compression.

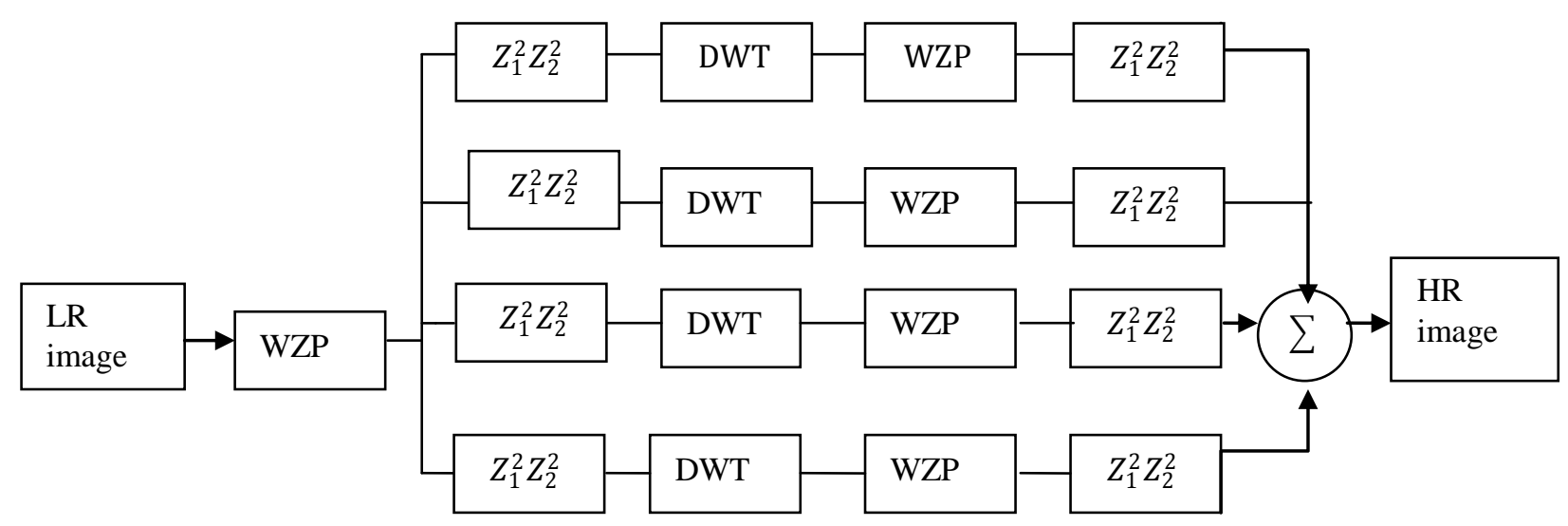

Fig 2.1 WZP- and CS-based image resolution enhancement. 


\section{Enhancement Of Satellite Image Resolution}

A discrete wavelet transform (DWT) is any wavelet transform for which the wavelets are discretely sampled. The Wavelet Series is just a sampled version of CWT and its computation may consume a significant amount of time and resources, depending on the resolution required. The Discrete Wavelet Transform (DWT), which is based on sub-band coding, is found to yield a fast computation of Wavelet Transform. It is easy to implement and reduces the computation time and resources required ${ }^{[5]}$. As with other wavelet transforms, a key advantage it has over Fourier transforms is temporal resolution: it captures both frequency and location information.

The transform of a signal is just another form of representing the signal. It does not change the information content present in the signal. The Wavelet Transform provides a time-frequency representation of the signal ${ }^{[8]}$. It was developed to overcome the shortcoming of the Short Time Fourier Transform (STFT), which can also be used to analyze non-stationary signals.

\subsection{Dwt-Based Resolution Enhancement}

DWT has been employed in order to preserve the high-frequency components of the image. DWT separates the image into different sub-band images, namely, LL, LH, HL, and HH. High-frequency sub-bands contain the high frequency component of the image ${ }^{[3]}$. The interpolation can be applied to these four sub-band images. In the wavelet domain, the low-resolution image is obtained by low-pass filtering of the high-resolution image. The low resolution image (LL sub-band), without quantization is used as the input for the proposed resolution enhancement process ${ }^{[9]}$.

In other words, low frequency sub-band images are the low resolution of the original image. Therefore, instead of using low-frequency sub-band images, which contains less information than the original input image, we are using this input image through the interpolation process. Hence, the input low-resolution image is interpolated with the half of the interpolation factor, $\alpha / 2$, used to interpolate the high-frequency sub-bands, as shown in fig 3.2. In order to preserve more edge information, i.e., obtaining a sharper enhanced image, we have proposed an intermediate stage in high frequency sub-band interpolation process.

As shown in Fig. 3.2, the low-resolution input satellite image and the interpolated LL image with factor 2 are highly correlated. The difference between the LL sub-band image and the low-resolution input image are in their high-frequency components. Hence, this difference image can be used in the intermediate process to correct the estimated high-frequency components. This estimation is performed by interpolating the high frequency sub-bands by factor 2 and then including the difference image (which is high-frequency components of low-resolution input image) into the estimated high-frequency images, followed by another interpolation with factor $\alpha / 2$ in order to reach the required size for IDWT process.

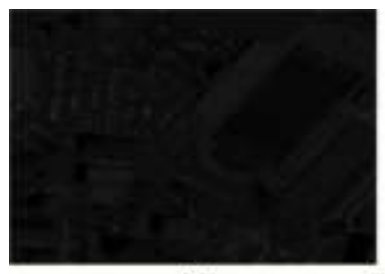

(a)

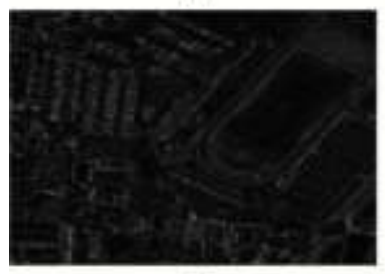

(c)

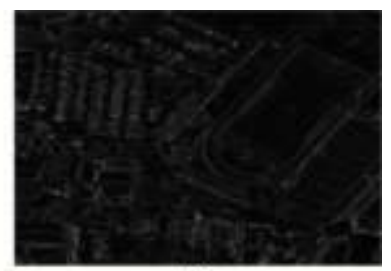

(b)

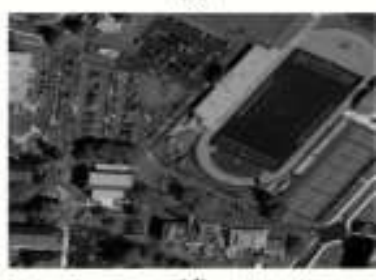

(d)

Fig.3.1 (a) The proposed enhanced image, (b) the standard bicubic interpolation, (c) The WZP- and CS-based image resolution enhancement technique and (d) the original high-resolution satellite image

Fig 3.1(a)-(c)show the difference between the high resolution images with the enhanced image by using the proposed resolution enhancement technique, the difference obtained by using bicubic interpolation directly, and the difference image with WZP and CS-based image resolution enhancement technique, respectively. Fig 3.1 (d) shows that higher-frequency components have been preserved in the proposed technique. 


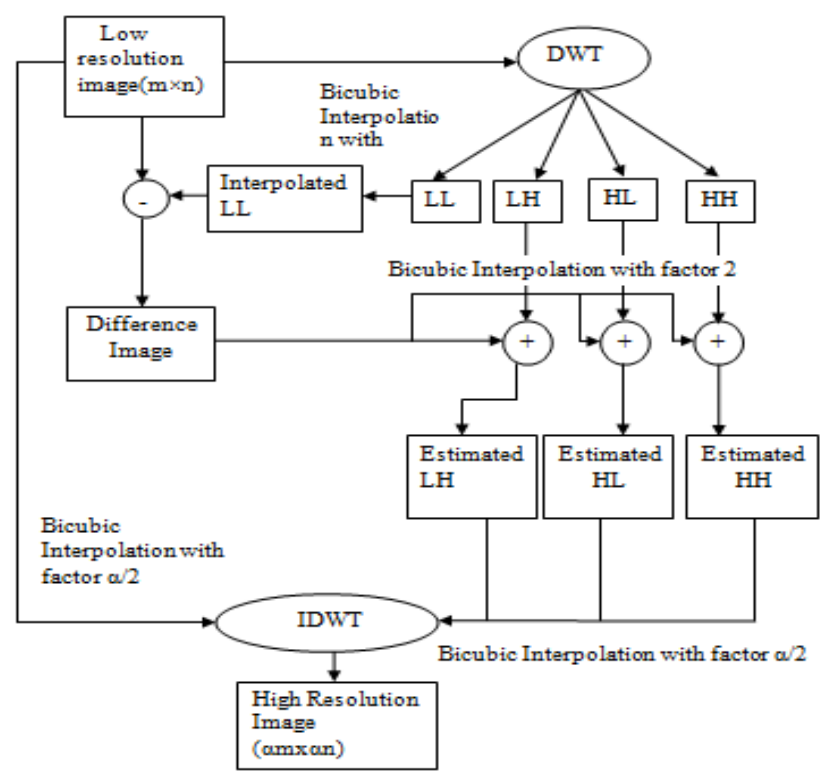

Fig 3.2 DWT resolution enhancement algorithm.

The intermediate process of adding the difference image, containing high-frequency components, generates significantly sharper and clearer final image. This sharpness is boosted by the fact that, the interpolation of isolated high-frequency components in $\mathrm{HH}, \mathrm{HL}$, and $\mathrm{LH}$ will preserve higher-frequency components than interpolating the low-resolution image directly.

Not only visual comparison but also quantitative comparisons is confirming the superiority of the proposed method. Peak signal-to-noise ratio (PSNR) and root mean square error (RMSE) have been implemented in order to obtain some quantitative results for comparison. PSNR can be obtained by using the following formula:

$$
P S N R=10 \log _{10}\left(\frac{R^{2}}{M S E}\right)
$$

Where $R$ is the maximum fluctuation in the input image ( 255 in here as the images are represented by 8 bits) and $M S E$ is representing the MSE between the given input image $\boldsymbol{I}_{\text {in }}$ and the original image $\boldsymbol{I}_{\text {org }}$ which can be obtained by the following:

$$
M S E=\frac{\sum_{i, j}\left(I_{i n}(i, j)-I_{\text {org }}(i, j)\right)^{2}}{M \times N}
$$

Where $M$ and $N$ are the size of the images.

Clearly, RMSE is the square root of the MSE, hence it can be calculated by the following:

$$
R M S E=\sqrt{\frac{\sum_{i, j}\left(I_{\text {in }}(i, j)-I_{\text {org }}(i, j)\right)^{2}}{M \times N}}
$$

\subsection{Results And Discussion}

\section{Implementation}

The implementation is done using MATLAB. The proposed resolution enhancement technique uses DWT to decompose the input image into different subband. Then, the high-frequency subband images and the input low-resolution image have been interpolated, followed by combining all these images to generate a new resolution-enhanced image by using inverse DWT. In order to achieve a sharper image, an intermediate stage (interpolation technique) has been used.

The discrete wavelet transform is applied to the input image and the four subband are obtained as shown in the Fig.3.3.

Bicubic interpolation of isolated high-frequency components in $\mathrm{HH}$, $\mathrm{HL}$, and LH will preserve higher-frequency components than interpolating the low-resolution image directly and they are shown in the Fig 3.4. 

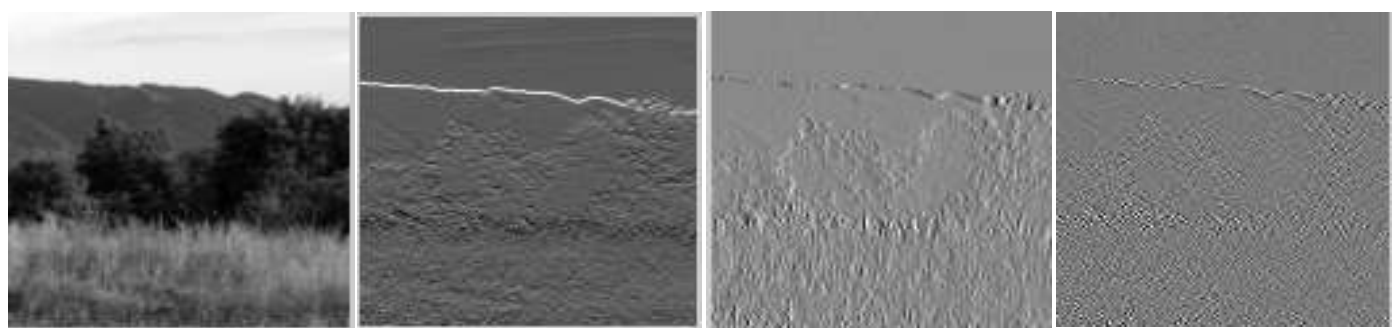

Fig 3.3 LL, LH, HL, HH subband of input image

Bicubic interpolation of isolated high frequency components in $\mathrm{HH}, \mathrm{HL}$, and $\mathrm{LH}$ will preserve higher-frequency components than interpolating the low-resolution image directly and they are shown in the Fig 3.4.

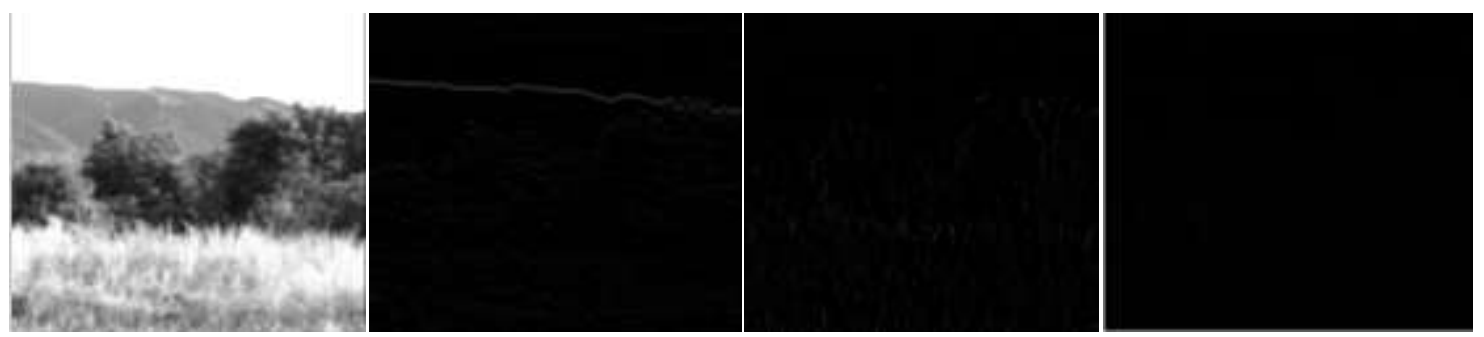

Fig 3.4 Bicubic interpolation of LL,LH,HL,HH subband.

The proposed technique has been tested on several different satellite images and they have superior resolution, smoother edges and sharper image over the conventional and state-of-art techniques from a visual point of view. The enhanced images by using bicubic interpolation, WZP and CS-based image resolution enhancement methods and the enhanced images obtained by the proposed technique are analyzed. It is clear that the resultant image, is sharper than the other techniques.

Table 1.1 PSNR, RMSE Results for DWT Method.

\begin{tabular}{|l|c|c|}
\hline IMAGE & RMSE & PSNR \\
\hline TEST & 3.79 & 37.8 \\
IMAGE & & 38.9 \\
\hline $\begin{array}{l}\text { MOON } \\
\text { IMAGE }\end{array}$ & 3.99 & 40 \\
\hline $\begin{array}{l}\text { WATER } \\
\text { IMAGE }\end{array}$ & 4.01 & \\
\hline
\end{tabular}

The PSNR improvement of the proposed technique is up to $7.19 \mathrm{~dB}$ compared with the standard bicubic interpolation. The PSNR and RMSE values are tabulated in the table 1.1. As expected, highest level of information content is embedded in the original images. The main reason of having the relatively high information content level of the images generated by the DWT technique is due to the fact that the unquantized input LL subband images contain most of the information of the original high-resolution image.

\subsection{SIMULATED OUTPUT}

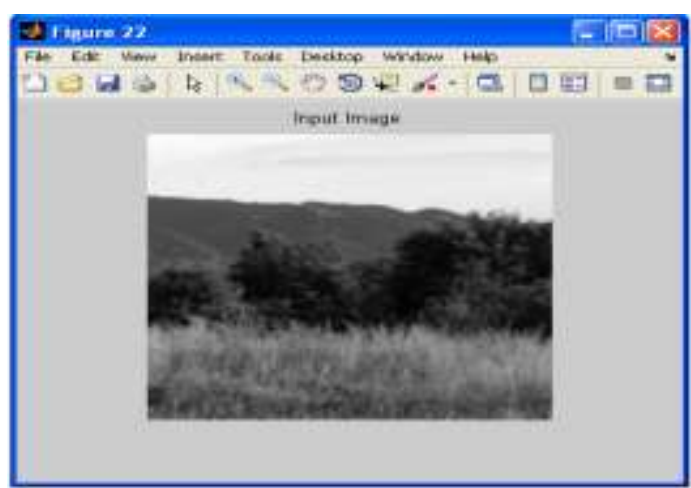

Fig4.1 Imput Image 


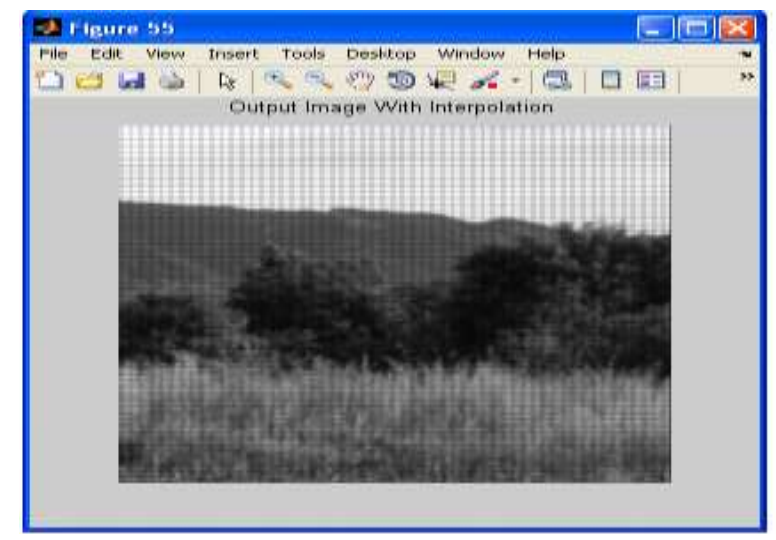

Fig 4.2 Output Image

\section{Conclusion \& Future Work}

This paper has proposed a new resolution enhancement technique based on the interpolation of the high-frequency subband images obtained by DWT and the input image. The proposed technique has been tested on well-known benchmark images, where their PSNR, RMSE and visual results show the superiority of the proposed technique over the conventional and state-of-art image resolution enhancement techniques. The PSNR improvement of the proposed technique is up to $7.19 \mathrm{~dB}$ compared with the standard bicubic interpolation.

For future work of the project may include color image enhancement instead of gray scale image enhancement. It also improves the efficiency of the input image by removal of noise. Also it may include the enhancement of satellite image with moving objects.

\section{References}

[1] G. Anbarjafari and H. Demirel, "Image super resolution based on interpolation of wavelet domain high frequency subbands and the spatial domain input image," ETRI J., vol. 32, no. 3, pp. 390-394, Jun. 2010.

[2] S.G Chang, Z. Cvetkovic and M. Vetterli, "Resolution enhancement of image using wavelet transform extrema ex-trapolation", Proc.ICASSP'95, vol.4,pp.2379-2382, May 1995

[3] M. S. Crouse, R. D. Nowak, and R. G. Baraniuk, "Wavelet-based statistical signal processing using hidden Markov models," IEEE Trans. SignalProcess., vol. 46, no. 4, pp. 886-902, Apr. 1998.

[4] H. Demirel, G. Anbarjafari, and S. Izadpanahi, "Improved motion-based localized super resolution technique using discrete wavelet transform for low resolution video enhancement," in Proc. 17th EUSIPCO, Edinburgh, U.K., Aug. 2009, pp. 1097-1101.

[5] H. Demirel and G. Anbarjafari, "Satellite image resolution enhancement using complex wavelet transform," IEEE Geosci. Remote Sens. Lett., vol. 7, no. 1, pp. 123-126, Jan. 2010.

[6] Glenn, W., Daniel, J.J., Zia, U.R. and Glenn, H. (2005) 'Enhancement of Imagery in Poor Visibility Conditions', Proceedings of SPIE, Vol. 5778, No.05, pp. 889.

[7] R. C. Gonzalez and R. E. Woods, Digital Image Processing. Englewood Cliffs, NJ: Prentice-Hall, 2007.

[8] HasanDemirel and GholamrezaAnbarjafari,'Discrete Wavelet Transform Based Satellite Image Resolution enhancement",IEEETrans.geosci.RemoteSens.,vol.49,no.6,pp.1997-2004,june 2011.

[9] H. Demirel, G. Anbarjafari, and S.Izadpanahi, "Improved motion-based localized super resolution technique using discrete wavelet transform for low resolution video enhancement," in Proc. 17th EUSIPCO, Edinburgh,U.K., Aug.2009, pp. 1097-1101.

[10] K. Kinebuchi, D. D. Muresan, and T.W. Parks, "Image interpolation using wavelet based hidden Markov trees," in Proc. IEEE ICASSP, 2001, vol. 3, pp. 7-11.

[11] N.G. Kingsbury. The dual-tree complex wavelet transform with improved orthogonality and symmetry properties. IEEE International Conference on Image Processing, pages 375-378, September 2000

[12] X. Li and M. T. Orchard, "New edge-directed interpolation," IEEE Trans. Image Process., vol. 10, no. 10, pp. 1521-1527, Oct. 2001.

[13] N. Nguyen, P. Milanfar, “An efficient wavelet-based algorithmfor image superresolution”, Proc. ICIP ‘00, vol.2, pp. 351-354, Sep. 2000

[14] Y. Piao, L. Shin, and H. W. Park, "Image resolution enhancement using inter-subband correlation in wavelet domain," in Proc. IEEE ICIP, 2007, vol. 1, pp. I-445-I-448.

[15] L. A. Ray and R. R. Adhami, "Dual tree discrete wavelet transform with application to image fusion," in Proc. 38th Southeastern Symp. Syst.Theory, Mar. 5-7, 2006, pp. 430-433.

[16] Temizel and T. Vlachos, "Wavelet domain image resolution enhancement using cycle-spinning," Electron. Lett., vol. 41, no. 3, pp. 119-121, Feb. 3, 2005.

[17] Waheeb, A.U., Akram, M. and Ziad, A.A. (2009) ‘ Gray Image Reconstruction', European Journal of Scientific Research, ISSN 1450-216X, Vol. 27 ,pp.167-173. 